\title{
Erratum: Spin Current Noise of the Spin Seebeck Effect and Spin Pumping [Phys. Rev. Lett. 120, 037201 (2018)]
}

\author{
M. Matsuo®, Y. Ohnuma®, T. Kato®, and S. Maekawa® \\ (2) (Received 4 August 2021; published 10 September 2021)
}

DOI: 10.1103/PhysRevLett.127.119902

In the discussion of the spin Hall angle in the Letter, the relation between $I_{C}^{\mathrm{ISHE}}$ and $I_{S}\left(\mathcal{S}_{C}^{\mathrm{ISHE}}\right.$ and $\left.\mathcal{S}\right)$ was incorrect. The correct relation is given as $I_{C}^{\mathrm{ISHE}}=\theta_{\mathrm{SH}} I_{S}\left(\mathcal{S}_{C}^{\mathrm{ISHE}}=\theta_{\mathrm{SH}}^{2} \mathcal{S}\right)$. Accordingly, Eq. (17) should be corrected as

$$
\theta_{\mathrm{SH}}=\frac{\mathcal{S}_{C}^{\mathrm{ISHE}} / I_{C}^{\mathrm{ISHE}}}{\mathcal{S} / I_{S}}
$$

We should also state that the form of the dynamic spin susceptibility for a dirty metal [1,2] used in our work includes a mistake as pointed out in recent theoretical works [3,4]. The main results of the Letter are unchanged even after replacing the dynamic spin susceptibility with the correct one, though the spin current and spin-current noise are quantitatively changed.

[1] H. Adachi, J.-I. Ohe, S. Takahashi, and S. Maekawa, Phys. Rev. B 83, 094410 (2011).

[2] M. Inoue, M. Ichioka, and H. Adachi, Phys. Rev. B 96, 024414 (2017).

[3] T. Kato, Y. Ohnuma, M. Matsuo, J. Rech, T. Jonckheere, and T. Martin, Phys. Rev. B 99, 144411 (2019).

[4] M. A. Silaev, Phys. Rev. B 102, 144521 (2020). 